\title{
Oléoprotéagineux : se démarquer par une démarche qualité
}

\author{
Céline Le Guillou ${ }^{1, *}$ et Valérie Duflot ${ }^{2}$ \\ 1 Terres Univia, 11 rue de Monceau, CS 60003, 75378 Paris cedex 08, France \\ 2 OCL, 66 rue la Boétie, 75008 Paris, France
}

Reçu le 23 novembre 2017 - Accepté le 11 mars 2018

La CLCV, l'une des associations françaises de consommateurs participant aux états généraux de l'alimentation en 2017, s'est montrée claire quant aux attentes des consommateurs: ils veulent de la qualité.

\section{Les 7 qualités que le consommateur est prêt à rétribuer}

La sensibilité des consommateurs au facteur qualité s'était beaucoup développée dans la fin des années 1990 et au début des années 2000. Dans l'enquête Tendances de consommation du CRÉDOC (Tavoularis et al., 2016), lorsqu'on demande aux consommateurs de choisir entre la qualité et le prix, le résultat est sans appel: en 2015 comme en 2000, ils déclarent privilégier la qualité quel que soit le secteur et à $78 \%$ pour les produits alimentaires. Ces dernières années cependant, la crise économique a induit une légère remontée de la sensibilité aux prix, sans pour autant supplanter la qualité.

Le CRÉDOC a identifié treize caractéristiques de qualité pour lesquelles le consommateur pourrait être prêt à payer plus cher. Sept sont citées par une très grande majorité d'enquêtés : la fiabilité (par 93\%), l'efficacité (92\%), le goût (91\%), la sécurité $(90 \%)$, la durée de vie $(87 \%)$, le respect de l'environnement $(87 \%)$ et la facilité d'utilisation $(81 \%)$. Viennent ensuite le soutien du commerce équitable $(75 \%)$, le gain de temps (67\%), l'avancée technologique $(58 \%)$ et la marque du produit $(50 \%)$. Enfin, le design (41\%) et la nouveauté $(35 \%)$ ne convainquent pas une majorité de consommateurs de payer plus pour de telles caractéristiques.

\section{Créer de la valeur ajoutée et sécuriser les débouchés}

Mieux répondre aux attentes «qualité » des consommateurs permet indubitablement de créer une valeur ajoutée et de se démarquer, évitant ainsi une compétition trop frontale avec les autres productions oléagineuses mondiales telles que le canola canadien ou encore le tournesol ukrainien. C'est pourquoi, les

*Correspondance : c.leguillou@terresunivia.fr acteurs de la filière ont développé une diversité de productions d'oléagineux de spécialité, permettant de créer davantage de valeur ajoutée et de sécuriser une partie des débouchés des producteurs : la filière oléique pour des qualités nutritionnelles et fonctionnelles reconnues, la filière érucique pour une diversité de débouchés en oléochimie, la filière Fleur de Colza et la filière bio. Dans chaque cas, la valorisation pour le producteur nécessite une démarche de filière avec l'accompagnement de l'ensemble des acteurs afin de valoriser l'origine française par la traçabilité, apporter conseil et formation aux agriculteurs, optimiser la supply chain, soutenir la recherche variétale et développer la contractualisation pluriannuelle (Tonin, 2018).

Plus récemment, en janvier 2016, producteurs, collecteurs, transformateurs, industriels se sont réunis au sein de Terres Univia pour former un groupe de travail sur le soja français. Son objectif : structurer de manière pérenne la filière soja sur le territoire français de la semence au produit soja vendu, tout en sécurisant ses capacités de production en amont comme en aval et en répondant aux demandes croissantes de qualité, de traçabilité et de durabilité. Pour cela, le groupe de travail a élaboré une charte qualité applicable à toutes les étapes : de la production de graines, à la $1^{\text {re }}$ utilisation ou transformation des graines de soja, en passant par la collecte. La charte qualité repose sur quatre engagements majeurs : (1) une origine France certifiée, (2) une culture et des produits non OGM, (3) une traçabilité garantie de la graine au produit transformé issu du soja, (4) des critères de durabilité. Cette démarche est en test sur la campagne 2017/2018 pour une mise en place réelle dès la campagne 2018/2019. L'ambition est d'atteindre 250000 hectares certifiés en 2025 ce qui permettrait de répondre à la demande des fabricants d'aliments pour le bétail en matières premières non OGM. En effet, la production actuelle de soja en France ne permet pas de satisfaire tous les besoins en tourteau de soja non OGM. Développer un soja origine France garanti non OGM permettra de le substituer à 500000 tonnes de tourteau d'importation non OGM.

\section{Se différencier par des logos officiels}

En France, plusieurs démarches visent à mettre en avant la qualité du produit. En premier lieu, l'utilisation des logos 
officiels (utilisables en France et en Europe) permet au consommateur de reconnaître les produits qui bénéficient d'un signe officiel d'identification de la qualité et de l'origine (SIQO). L'INAO est chargé de la supervision du dispositif de contrôle pour l'ensemble des signes officiels de qualité et d'origine. Il agréé et évalue les organismes indépendants chargés du contrôle du respect des cahiers des charges. Á ce jour, il existe 5 types de SIQO: les AOC/AOP (Appellation d'origine contrôlée en France, Appellation d'origine protégée en Europe), les IGP (Indication géographique protégée), l'AB (Agriculture biologique), Le Label Rouge et les spécialités traditionnelles garanties. Ces SIQO suivent trois grands principes: (1) une démarche collective et volontaire émanant de producteurs ou d'un groupement de producteurs, (2) Des conditions de production strictes validées par l'État, (3) Des contrôles réguliers réalisés par des organismes indépendants agréés par l'État. Ces SIQO sont pour les consommateurs une garantie officielle de l'origine (AOC et AOP; IGP), de la qualité supérieure (Label rouge), d'une recette traditionnelle (STG) et/ou respect de l'environnement (Agriculture biologique).

Les huiles d'olive ont rapidement saisi l'intérêt de ces logos officiels pour se différencier: dès 1994, la première appellation d'origine oléicole a vu le jour. Une naissance qui en a appelé d'autres, bénéficiant du et au dynamisme de la filière oléicole. En 2018, les huiles françaises comptent 8 appellations d'origine: Nyons, Vallée des Baux-de-Provence, Aix-enProvence, Haute-Provence, Nice, Nîmes, Corse-Oliu di Corsica et Provence (Paris, 2018).

\section{Se différencier par une démarche privée}

En parallèle, coexistent des démarches plutôt privées, émanant d'une filière ou d'un industriel. En 2000, est par exemple née l'association Bleu Blanc Cœur (BBC) qui regroupe l'ensemble des acteurs de la chaîne alimentaire autour d'un objectif commun de qualité, que ce soit dans le domaine animal, environnemental ou de l'alimentation humaine. Les fondamentaux de BBC sont les suivants: garantir une chaîne alimentaire respectée du champ à l'assiette, bien nourrir les hommes, bien nourrir les animaux, préserver l'environnement. BBC a conduit cinq études cliniques chez l'homme mettant en évidence le rôle de l'alimentation sur la santé du consommateur. La première a été publiée en 2002 dans Annals of Nutrition and Metabolism (Weill et al., 2002). Dans notre dossier, l'équipe de Bleu Blanc Cœur publie un article montrant qu'un menu BBC consommé tous les 15 jours (représentatif de la consommation moyenne pour la population française des hommes adultes) permet de réduire efficacement l'écart entre consommation réelle des Français et les apports conseillés en acides gras (comparativement à un menu élaboré en utilisant des produits animaux issus de modes de production standard) (Schmitt et al., 2017). Rappelons d'ailleurs que tout produit (lait, œuf, jambon...) suivant le cahier des charges BBC peut apposer le logo sur son emballage.

Les exigences multiples du consommateur d'aujourd'hui guident une multitude d'autres démarches privées:

- le développement de l'agriculture biologique et une demande grandissante pour une alimentation plus naturelle incitent l'industrie à revisiter les procès mis en œuvre dans l'extraction des huiles végétales : pariant sur le dévelop- pement de solutions d'extraction sans hexane, un nouveau procédé basé sur l'utilisation d'éthanol au-dessus du point d'ébullition est en cours d'évaluation économique (Carré et al., 2018); l'extraction éthanol suivie par la démixtion à basse température du miscella permet d'obtenir une qualité de l'huile élevée, notamment exempte d'acides gras libres et de phospholipides (Citeau et al., 2018);

- la sensibilité grandissante du consommateur à l'écologie explique la mise en place de l'outil ACéVOIL d'évaluation des impacts environnementaux des produits alimentaires formulés à partir d'huiles végétales. Il fournit des résultats communicables aux clients et/ou consommateurs du produit afin, notamment, de se différencier des produits concurrents et de valoriser les actions d'écoconception mises en œuvre (Badey et Bosque, 2018);

- la volonté des agriculteurs de faire découvrir leur quotidien, leur métier et leur savoir-faire a donné naissance en 2015 à la marque Terres OléoPro. Son objectif: identifier les plantes riches en huiles ou en protéines dans les produits de notre quotidien. Les cinq engagements garantis par le cahier des charges Terres OléoPro sont: (1) l'origine française de nos cultures, (2) les bonnes pratiques agricoles et industrielles, (3) le respect de la biodiversité et la réduction des impacts sur l'environnement, (4) le progrès continu et l'innovation, (5) l'engagement social et sociétal.

\section{Au-delà de nos frontières}

Cette démarche de différenciation dépasse bien entendu les frontières de l'hexagone et même de l'Europe comme en témoigne le «projet arganier» qui a, entre autres, aboutit à l'obtention d'une IGP mais aussi mélangé harmonieusement le développement durable, la recherche-action intégrée et le progrès économique et social (Charrouf et Guillaume, 2018).

En Afrique également, le développement de productions locales permettant de réduire l'insécurité alimentaire, d'améliorer le revenu rural, de favoriser des emplois pérennes et la mise en place de systèmes agricoles et alimentaires durables et résilients explique la relance des oléagineux au Maroc dans le contexte du Plan Maroc Vert, et le projet de développement du soja au Burkina-Faso porté par Agropol (Le Guilloux et al., 2018).

\section{Quelle visibilité pour le consommateur?}

Reste à savoir comment le consommateur s'y retrouve dans ce contexte de profusion des signes de qualité : la coexistence de deux signes de qualité sur un même produit a-t-elle des conséquences sur la valeur de chaque signe? Des éléments de réponses sont livrés par Sylvette Monier-Dilhan (MonierDilhan, 2018).

\section{Références}

Badey L, Bosque F. 2018. Product environmental assessment as vector of differentiation-ACéVOIL, a new tool for the vegetable oil sector. OCL 25: D208. 
Carré P, CiteauM, Dauguet S. 2018. Hot ethanol extraction: economic feasibility of a new and green process. OCL 25: D206.

Charrouf Z, Guillaume D. 2018. The argan oil project: going from utopia to reality in 20 years. OCL 25: D209.

Citeau M, Albe Slabi S, Joffre F, Carré P. 2018. Improved rapeseed oil extraction yield and quality via cold separation of ethanol miscella. $O C L$ 25: D207.

Le Guilloux G, David A, Pouzet A. 2018. La différenciation par l'organisation de filières : l'expérience d'AGROPOL. OCL 25: D210.

Monier-Dilhan S. 2018. Food labels: consumer's information or consumer's confusion. OCL 25: D202.

Paris A. 2018. L'huile d'olive française : les appellations d'origine en question. OCL 25: D204.
Schmitt B, Ferry C, Mairesse G, et al. 2017. The choice of animal feeding system influences fatty acid intakes of the average French diet. OCL 25: D205.

Tavoularis G, Hébel P, Billmann M, Lelarge C. 2016. Les Français toujours très attachés à la qualité. Consommation et mode de vie, $\mathrm{n}^{\circ}$ 283. ISSN 0295-9976. http://www.credoc.fr/pdf/4p/283.pdf (dernière consult. mars 2018).

Tonin P. 2018. Les productions françaises d'oléagineux de spécialité : des démarches en filière pour créer de la valeur dans nos territoires. OCL 25: D203.

Weill P, et al. 2002. Effects of Introducing linseed in livestock diet on blood fatty acid composition of consumers of animal products. Ann Nutr Metab 46:182-191.

Citation de l'article : Le Guillou C, Duflot V. 2018. Oléoprotéagineux : se démarquer par une démarche qualité. OCL 25(2): D201. 